\title{
Channel Coding for Multimedia Transmission on High-Speed Flying Devices
}

\author{
Sulthon Muhammad Fauzi Aulia ${ }^{\mathrm{a}, \mathrm{b}, 1}$, Khoirul Anwar ${ }^{\mathrm{a}, \mathrm{b}, 2}, \mathrm{Nur}$ Andini ${ }^{\mathrm{b}, 3}$ \\ ${ }^{a}$ The University Center of Excellence for Advanced Intelligent Communications (AICOMS), \\ ${ }^{b}$ School of Electrical Engineering, Telkom University, Bandung, 40257, Indonesia \\ Corresponding author: ${ }^{1}$ smfauzia@student.telkomuniversity.ac.id, ${ }^{2}$ anwarkhoirul@telkomuniversity.ac.id, \\ ${ }^{3}$ nurandini@telkomuniversity.ac.id
}

\begin{abstract}
Communication systems for high-speed flying devices, such as drones and missiles, have performances with error-floor caused by the Doppler effect, which causes inter-carrier interference (ICI) and destroys real-time data transmission. Channel coding cannot reduce error-floors, but channel coding may still achieve performance with turbo-cliff. This paper proposes a broadband communication system for high-speed flying devices using soft 4 quadrature amplitude modulation (4-QAM) modulations with the optimal threshold $\mathcal{S}$ for practical implementation assuming that the maximum/minimum log-likelihood ratio (LLR) values of \pm 709 . We use orthogonal frequency division multiplexing (OFDM) with low-density parity-check (LDPC) codes as the channel coding scheme and minimum mean squared error (MMSE) equalization. To reduce the computational complexity and to keep the data rate high, we use only a single pilot for the channel estimation. Computer-based simulations for several high speeds are performed to evaluate the performance of the proposed high-speed flying devices system. The bit error rate (BER) performance is evaluated based on LLR under additive white Gaussian noise (AWGN) and multipath Rayleigh fading channels. The results confirmed that the proposed system with the optimal threshold $\mathcal{S}$ can avoid unstable jumping error with better turbo-cliff and lower error-floor. The maximum speed the system can achieve for BER of $10^{-2}$ is $400 \mathrm{~km} / \mathrm{h}$. The results of this paper are expected to contribute significantly to the development of communication systems on flying devices.
\end{abstract}

Keywords- Doppler effect; OFDM; LDPC; real-time; BER; soft demapper.

Manuscript received 4 Jun. 2021; revised 19 Jul. 2021; accepted 18 Aug. 2021. Date of publication 31 Aug. 2021. IJASEIT is licensed under a Creative Commons Attribution-Share Alike 4.0 International License.

\section{INTRODUCTION}

Wireless communication is widely used for communication systems on mobile devices, such as high-speed flying devices like drone or missile. However, movement in a wireless communication system can cause the Doppler effect. The Doppler effect causes the frequency shifting, causing intercarrier interference (ICI), which is damaging the data. The frequency shift depends on the relative speed of the transmitter and receiver of the devices and the carrier frequency used. The greater carrier frequency and speed can make the shift of the frequency also greater, and vice versa [1]. The Doppler effect also causes channels to be timevarying or time-selective fading, i.e., channel that change rapidly time-by-time [1]. If the rapid change to this channel cannot be captured at the receiver, an error-floor appears. The error-floor appears because the captured signals are equalized using the wrong channel.

Some high-speed flying devices can receive and transmit multimedia data in real-time by utilizing broadband wireless communication systems, which have high data-rate. 


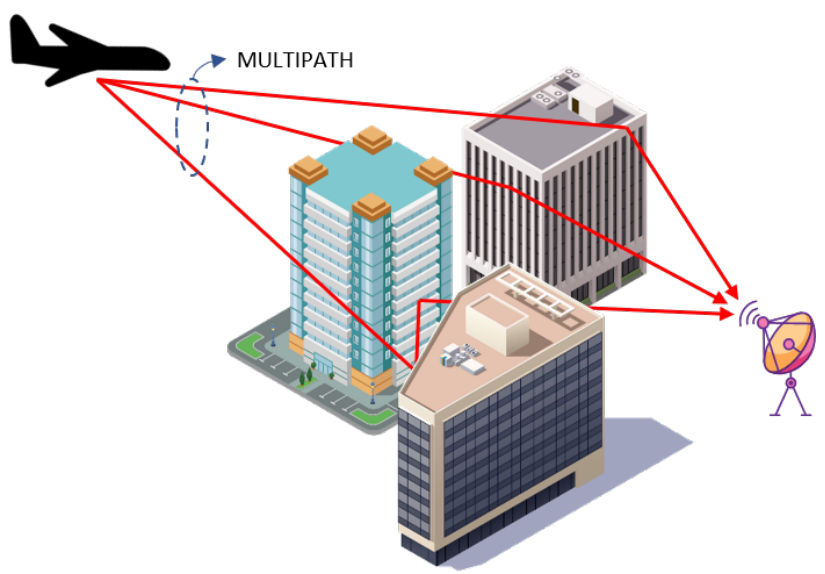

Fig. 1 Illustration of communication between the transmitter and the receiver using bradband comunication through multipath Rayleigh fading.

Broadband communication systems transmit data in multiple paths or multipath. However, the problem with multipath data transmission is multipath fading as shown in Fig. 1. Diffracted waves can also cause the waves that arrive late. These late waves can interfere with the other waves or vice versa, called inter-symbol interference (ISI) [2].

The multipath fading can be slightly overcome by parallel transmission. Orthogonal frequency division multiplexing (OFDM) is the most implemented multicarrier transmission scheme [3]. OFDM is widely used for digital transmission because it can correct errors due to multipath fading [4]. The use of the OFDM technique can still produce errors because OFDM is vulnerable to frequency shifts caused by the Doppler effect and phase noise called ICI [5] and causes error-floor. The uses of channel coding cannot reduce errorfloors, but channel coding may still achieve performance with turbo-cliff. Channel coding has various types, one of which is the low-density parity-check (LDPC) codes. Robet Gallager invented LDPC codes in his PhD thesis in 1962 [6]. After 30 years of neglect due to the lack of computing power, MacKay and Neal developed the LDPC codes [7].

LDPC codes is chosen because it has a better performance than Turbo codes which is known to have a performance close to Shannon limit [8]. LDPC is also widely used for high-throughput and real-time communications [8]. LDPC also has a good ability on moderate $(\sim 400)$ and large block size $(\sim 6144)$ [9]. To achieve bit error rate (BER) performances that are close to channel capacity, the LDPC codes must have thousands of bits of LDPC codes block length $N_{\text {LDPC }}[10]$.

We consider using LDPC codes with the structure of the second generation digital terrestrial television broadcasting system (DVB-T2) because this flying devices also have functions for sending and receiving multimedia data. However, DVB-T2 LDPC codes has a block length $N_{\text {LDPC }}=16200$ and $N_{\mathrm{LDPC}}=64800$ and requires a long process and high complexity in the encoding and decoding process. Because flying devices has limited battery lifetime, the communi- cation system requires low computational complexity of the encoding and decoding process. Therefore, we consider downscaled DVB-T2 LDPC codes, which has a short block length. In research [11], they simplified the DVB-T2 LDPC codes with downscaling technique from $N_{\mathrm{LDPC}}=16200$ to $N_{\text {LDPC }}=270$.

However, some devices have limitations in performing calculations. This limitation causes the LLR to be infinity and unstable jumping error. In [12], Hamdi et al. researched and proposed the best $d_{p}$ for fifth generation new radio (5GNR) [12] communication to solve this problem.

In this paper, we proposes a broadband communication system for high-speed flying devices using soft 4 quadrature amplitude modulation (4-QAM) modulations with the optimal threshold $\mathcal{S}$ for practical implementation helped by DVB-T2 LDPC codes. The optimal threshold $\mathcal{S}$ is used to avoid the unstable jumping error with better turbo-cliff and lower error-floor.

The rest of this paper is organized as follows: Section II describes the materials and method that used in this paper. Section III describes the performance of the proposed system. Section IV concludes the paper.

\section{MAterials And Method}

\section{A. System Model}

Fig. 2 shows a communication system model on a highspeed flying devices from the transmitter $\left(T_{x}\right)$ to the receiver $\left(R_{x}\right)$. We used downscaled DVB-T2 LDPC codes from [11] for the channel coding. We also used 4 quadrature amplitude modulation (4-QAM) modulation and OFDM as the transmission scheme.

Fig. 2 shows information source $b$ generated randomly. The bits are encoded by block "C" into block "V" with block interleaver $\Pi_{x}$. The encoding process changes the bit information $b$ to codeword $c$. The codeword enters the modulation process using the 4-QAM modulator "M", and forms a symbol $\chi$. 4-QAM generates a modulation symbol that has a pair of information bits in each symbol $\chi$ [13]. After the symbol is modulated, the symbol is transformed from frequency domain to time domain using block "IFFT" and produces symbol $\varkappa$. The block "CP" function is adding a cyclic prefix (CP), forms a symbol $\mathrm{x}$. Then, The symbols are sent through the channel. This paper uses the additive white Gaussian noise (AWGN) and multipath Rayleigh fading channel models.

Received information symbol $\mathbf{y}$ experiences channel distortion and defines

$$
\mathbf{y}=h \cdot \mathbf{x}+\mathbf{n},
$$

where $\mathbf{n}$ is noise vector, $h$ is 1 for the AWGN channel and the random complex number for the multipath Rayleigh fading channel which can be expressed by

$$
h=\frac{\mathcal{A}+j \cdot \mathcal{B}}{\sqrt{2}} ; \mathcal{A} \sim \mathcal{N}\left(0, \sigma^{2}\right) ; \mathcal{B} \sim \mathcal{N}\left(0, \sigma^{2}\right),
$$

where $\mathcal{A}$ and $\mathcal{B}$ are each normally distributed random numbers with zero mean and $j=\sqrt{-1}$. The blok "CP 


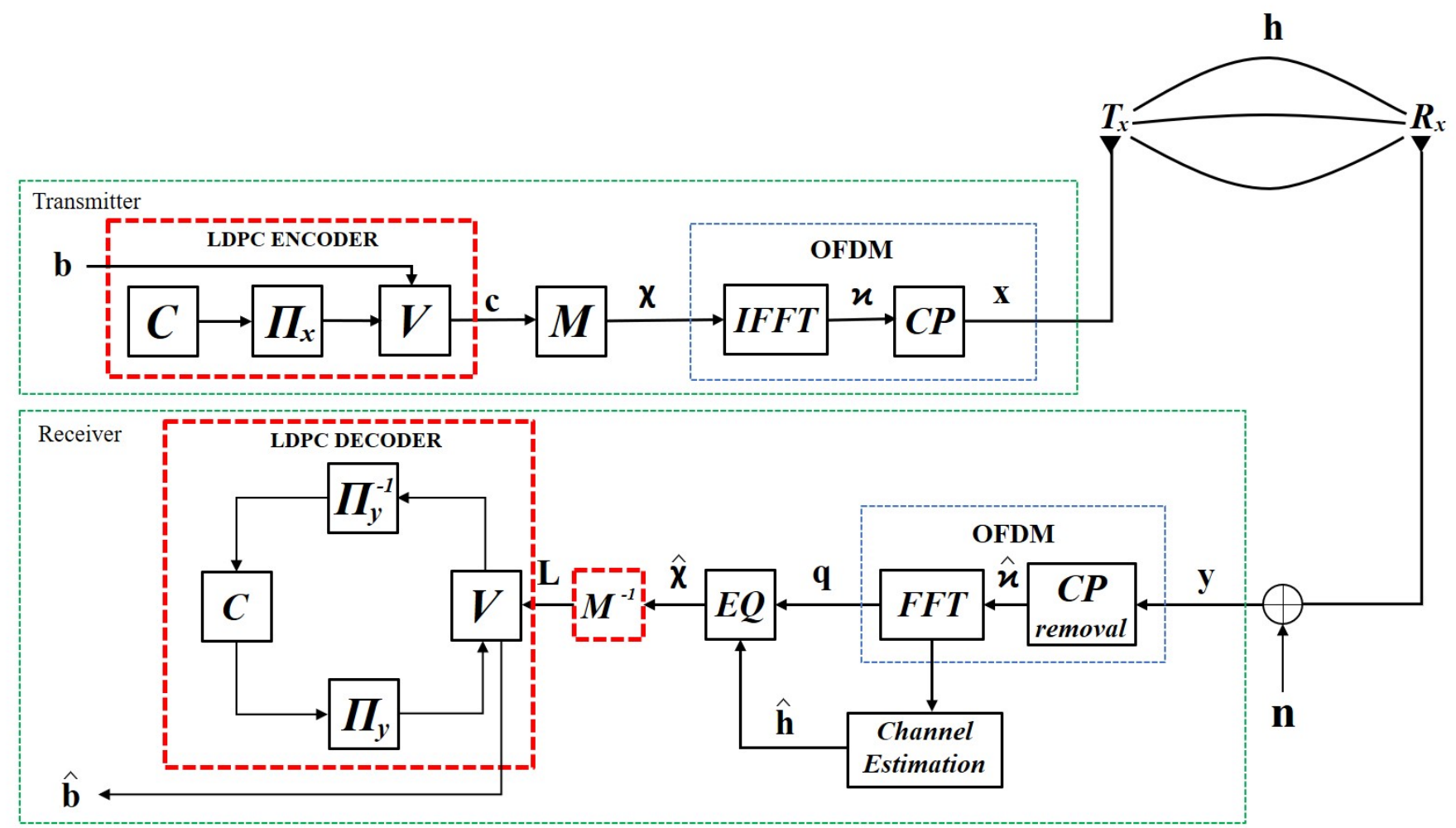

- The proposed section in this paper

Fig. 2 System model of OFDM for high-speed flying devices.

removal" function is removing $\mathrm{CP}$ that has been added in the symbol $\mathbf{y}$ from block "CP" and forms a new symbol $\hat{\varkappa}$. The symbol $\hat{\varkappa}$ can be expressed by

$$
\hat{\varkappa}=\mathbf{H}_{c} \cdot \varkappa+\mathbf{n},
$$

where $\mathbf{H}_{c}$ is circulant matrix.

Then, the symbol $\hat{\varkappa}$ is transformed using the block "FFT" to return the symbol to the frequency domain and create the symbol q before entering the equalizer block "EQ". Symbol q can be expressed by

$$
\mathbf{q}=\mathbf{F} \cdot \mathbf{H}_{\mathbf{c}} \cdot \mathbf{F}^{H} \cdot \chi+\mathbf{n},
$$

where $\mathbf{F}$ is fast Fourier transform (FFT) matrix, $\mathbf{F}^{\mathbf{H}}$ is inverse fast Fourier transform (IFFT) matrix.

In the block "channel estimation", channel estimation is carried out using the pilot-assisted channel estimation technique, which produces $\hat{h}$ and become an input for the block "EQ". Furthermore, the equalization process on the equalizer uses minimum mean squared Error (MMSE) to produce the output $\hat{\chi}$ symbol for the equalizer. The $\hat{\chi}$ symbol can be expressed by

$$
\hat{\chi}=\mathbf{q} \cdot w,
$$

where $w$ is coefficient of the equalizer and it can be expressed by

$$
w=\frac{\psi^{*}}{\psi \psi^{*}+\sigma^{2}},
$$

where $\hat{\psi}^{*}$ is complex conjugate of $\psi$ and $\psi$ can be expressed by

$$
\psi=\operatorname{diag}\left[\mathbf{F} \cdot \mathbf{H}_{\mathbf{c}} \cdot \mathbf{F}^{H}\right],
$$

This paper uses a soft decoding technique as decoding technique. The $\hat{\chi}$ symbol is demodulated with the 4-QAM demodulator " $\mathrm{M}^{-1}$ " to create the log-likelihood ratio (LLR) $L$ and be the input for the LDPC codes decoder. The iterative decoding process in the LDPC decoder is carried out by block "V", deinterleaver block $\Pi_{y}^{-1}$, block "C", and interleaver block $\Pi_{y}$.

The method of decoding LDPC codes uses the sumproduct algorithm (SPA) algorithm. This step aims to return the $L$ in the form of information bits $\hat{b}$ and determine whether the received bits are the same as the transmitted bits, then the BER is obtained. BER calculation $P_{b}$ in AWGN with 4-QAM can be expressed with [2]

$$
P_{b_{4-\mathrm{QAM}-\mathrm{AWGN}}}=\frac{1}{2} \operatorname{erfc}\left(\sqrt{E_{b} / N_{0}}\right),
$$

where $E_{b} / N_{0}$ is normalized signal-to-noise ratio (SNR). BER calculation $P_{b}$ in multipath Rayleigh fading with 4QAM can be expressed with [2]

$$
P_{b_{4-\mathrm{QAM}-\mathrm{fading}}}=\frac{1}{2}\left[1-\frac{1}{\sqrt{1+\frac{1}{E_{b} / N_{0}}}}\right] .
$$




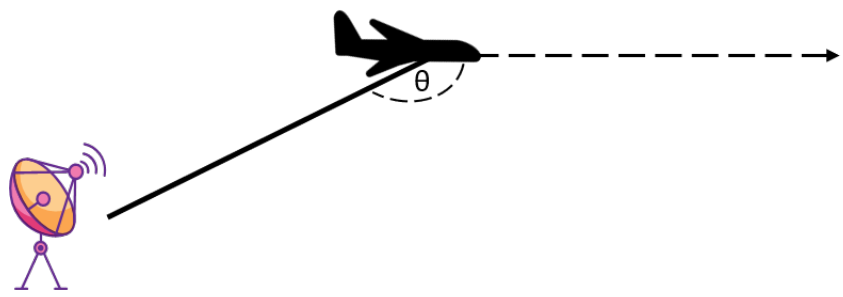

Fig. 3 The illustration of the angle between the transmitter and the receiver that causes the Doppler shift.

\section{B. Doppler Effect}

The Doppler effect occurs when a transmitter or receiver experiences movement at a relative speed which causes the frequency shift. The frequency shift that happens $f_{d}$ can be calculated by

$$
f_{d}=\frac{v \cos \theta}{c} f_{c}
$$

where $v$ is the relative velocity $(\mathrm{m} / \mathrm{s}), c$ is the speed of light $(\mathrm{m} / \mathrm{s}), f_{c}$ is the carrier frequency $(\mathrm{Hz})$, and $\theta$ is the angle between the transmitter and receiver. The shift also depends on the size of $\theta$. If the $\theta$ is between $0^{\circ}$ and $90^{\circ}$, the received frequency is positive and greater than the carrier frequency. When the $\theta$ and between $90^{\circ}$ and $180^{\circ}$, the received frequency is negative and smaller than the carrier frequency, as shown in Fig. 3.

The Normalized Doppler Spread on OFDM symbol duration is $f_{d} T_{s} . F_{d} T_{s}$ can be calculated by

$$
f_{d} T_{s}=f_{d} \cdot T_{s},
$$

where $T_{s}$ is symbol duration and can be obtained by

$$
T_{s}=\frac{1}{B_{s c} / \kappa},
$$

where $B_{s c}$ is subcarrier spacing and $\kappa$ is sample. Then, $f_{d} T_{s}$ can be expressed by

$$
f_{d} T_{s}=\frac{f_{d}}{B_{s c} / \kappa}
$$

\section{Low-Density Parity-Check (LDPC) Codes}

The form of the parity check matrix matrix $\mathbf{H}$ LDPC codes are adjusted to the number of column $(N)$ in matrix $\mathbf{H}$ equals to codeword $c$, the number of row $(K)$ in matrix $\mathbf{H}$ equals to messages bits $b$, redundancy bit $(M)$, degree of check node $\left(d_{c}\right)$ (i.e., the number of " 1 " in one column of the matrix), and the degree of variable nodes $\left(d_{v}\right)$ (i.e., the number of " 1 " in a row of the matrix) with

$$
M=N-K,
$$

then matrix $\mathbf{H}$ has the dimensions $M \times N$. Each row in matrix $\mathbf{H}$ represents a Check Nodes $(\mathrm{CN})$ and each column in $\mathbf{H}$ represents a Variable Nodes (VN).

The encoder uses the Generator Matrix G LDPC codes to create the codeword. The matrix $\mathbf{H}$ is created based on

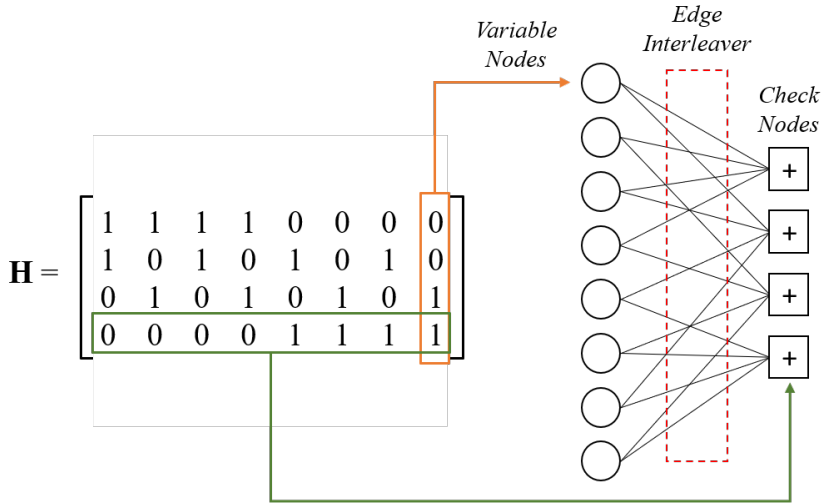

Fig. 4 Example of parity-check matrix regular $\operatorname{LDPC}(2,4)$ and corresponding tanner graph.

the matrix $\mathbf{H}$ by performing the Gauss-Jordan elimination operation on the matrix $\mathbf{H}$ to form [14]

$$
\mathbf{H}=\left[\begin{array}{ll}
\Delta & I_{N-K}
\end{array}\right],
$$

where $\Delta$ is binary matrix $(N-K) \times K$ and $I_{N-K}$ is identity matrix with order $N-K$. If the form of the matrix $\mathbf{H}$ has been obtained, then the matrix $\mathbf{H}$ is obtained with form [14]

$$
\mathbf{G}=\left[\begin{array}{ll}
I_{N-K} & \Delta^{T}
\end{array}\right]
$$

where $\Delta^{T}$ is transpose $\Delta$. Then, the codeword $c$ is obtained with

$$
c=b \cdot \mathbf{G} \text {. }
$$

LDPC codes come in two forms. Regular LDPC codes have a constant $d_{c}$ and $d_{v}$ in each row and column [15]. Irregular LDPC codes have a varied $d_{c}$ and $d_{v}$ in each row and column [15]. Irregular LDPC codes also have higher girth than Regular LDPC codes. It makes Irregular LDPC codes have better performance than regular LDPC codes.

The matrix $\mathbf{H}$ can be represented in a bipartite graph with a Tanner graph [16]. Tanner graph has an edge interleaver that connects $\mathrm{VN}$ and $\mathrm{CN}$, if and only if $\mathrm{VN}$ and $\mathrm{CN}$ have a relationship that is indicated by a non-zero on the matrix, as shown in Fig. 4. VNs and CNs are connected if the element of matrix $\mathbf{H}$ is "1".

\section{DVB-T2 LDPC codes}

Based on [17], the parity check matrix H DVB-T2 LDPC codes has $N_{\mathrm{LDPC}}=16200$ and $N_{\mathrm{LDPC}}=64800$ that has the following characteristics:

- The information section has a cyclic structure. This characteristic can be implemented in encoders and decoders based on partly parallel processing architecture

- The parity section has a staircase structure. This characteristic can be used to form parity bits with an accumulator.

DVB-T2 LDPC codes has code rates effective $R_{e}=$ $\left\{\frac{4}{9}, \frac{3}{5}, \frac{2}{3} \frac{11}{15}, \frac{7}{9}, \frac{37}{45}\right\}$. The matrix $\mathrm{H}$ has a dimension that can be determined by

$$
\left(1-R_{e}\right) \cdot N \times N
$$


TABLE I

THE MATRIX H DIMENSIONS AND BITS OF INFORMATION CAN BE SENT FOR EACH CODE RATES.

\begin{tabular}{llcc}
\hline \multicolumn{1}{c}{$\mathbf{R e}$} & \multicolumn{1}{c}{$\mathbf{K}$} & \multicolumn{1}{c}{$\mathbf{N}$} & $\mathbf{b}$ \\
\hline $4 / 9$ & 150 & 270 & 120 \\
$3 / 5$ & 108 & 270 & 162 \\
$2 / 3$ & 90 & 270 & 180 \\
$11 / 15$ & 72 & 270 & 198 \\
$7 / 9$ & 60 & 270 & 210 \\
$37 / 45$ & 48 & 270 & 222 \\
\hline
\end{tabular}

The matrix $\mathbf{H}$ can also be represented by

$$
\mathbf{H}=\left[\begin{array}{ll}
\mathbf{H}_{\mathbf{a}} & \mathbf{H}_{\mathbf{b}}
\end{array}\right],
$$

where each dimension matrix $\mathbf{H}_{\mathbf{a}}$ is $(N-K) \times K$, and matrix $\mathbf{H}_{\mathbf{b}}$ matrix is $K \times K$. The matrix $\mathbf{H}_{\mathbf{a}}$ contains bits of information formed based on the Addresses of Parity Bit Accumulators table for each $R_{e}$, and the matrix $\mathbf{H}_{\mathrm{b}}$ contains parity bits in the form of a staircase matrix. Each $R_{e}$ matrix $\mathbf{H}$ can send information bits $b$ as much as

$$
b=N-K \text {. }
$$

In this paper, we consider use downscaled DVB-T2 LDPC codes from $N=16200$ to $N=270$ [11]. Downscaled DVBT2 LDPC codes is used because it has lower complexity than LDPC $N=16200$. This is intended to speed up the encoding and decoding process. Table I shows the dimensions and bits of information that can be sent for each $R_{e}$.

Degree distributions of downscaled DVB-T2 LDPC codes is denoted by $\Lambda(x)$ and $\Omega(x)$ for each variable node degree (VND) and check node degree (CND). The degree distributions are used by this paper for each code rates are same like the degree distributions that used in [11].

\section{E. Sum-Product Algorithm (SPA)}

SPA has better performance compared to other decoding techniques in LDPC codes [18]. SPA works on connected VNs and CNs by sending messages over the connection [19]. Each VNs sends LLR to the connected CNs. CNs process the LLR that the connected VNs have sent, and the CNs can predict each VN. Each connected VN process and add all the predicted LLR by CNs to produce a more precise LLR. This is the first iteration. This iteration keep continues until it meets the stop criteria until the LLR for each VN approaches " +1 " or " $-1 "$.

The output of each VNs and CNs can be called extrinsic LLR $L_{E}$ as shown in Fig. 5. $L_{E_{v}}$ is from VNs and $L_{E_{c}}$ is from CNs. After interleaver or deinterleaver, $L_{E}$ is sent to VNs or CNs as a priori LLR $L_{A} . L_{A_{v}}$ is sent to VNs and $L_{A_{c}}$ is sent to CNs.

The operation in $\mathrm{VN}$ as shown in Fig. 6(a) can be expressed by [11]

$$
L_{E_{v_{j}}}(k)=L+\sum_{i=1, i \neq k}^{d_{v_{k}}} L_{A_{v_{i}}},
$$

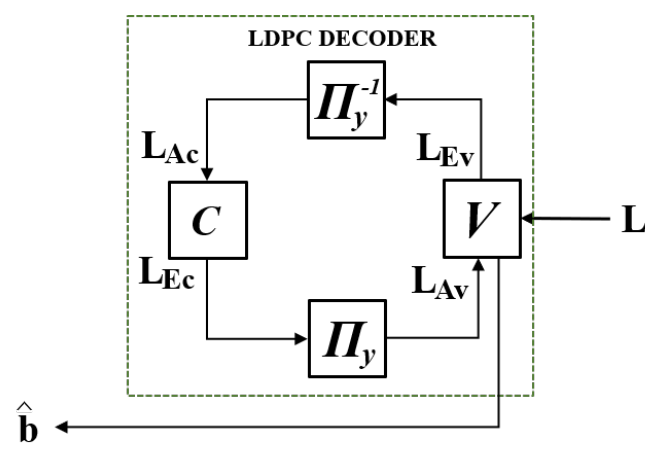

Fig. 5 Block diagram of the LDPC decoder.
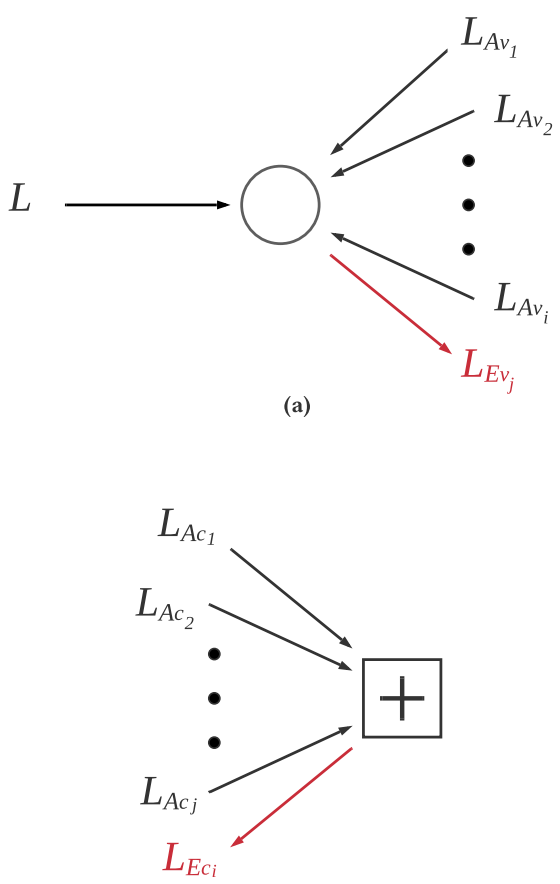

(b)

Fig. 6 Message flow in: (a) Variable Nodes and (b) Check Nodes.

in the first iteration, the $L_{A_{v}}$ is 0 . Then the operation in $\mathrm{CN}$ as shown in Fig. 6(b) can be expressed by [11]

$$
L_{E_{c_{i}}}(n)=\sum_{j=1, j \neq n}^{d_{c_{n}}} \boxplus L_{A_{c_{j}}},
$$

but (22) can be simplified by some mathematical manipulation, and can be written as [19]

$$
\begin{aligned}
L_{E_{c_{i}}}(n) \approx & \operatorname{sgn}\left\{L_{A_{c_{j}}}\right\} \cdot \operatorname{sgn}\left\{L_{A_{c_{j+1}}}\right\} \\
& \cdot \min \left\{\left|L_{A_{c_{j}}}\right|,\left|L_{A_{c_{j+1}}}\right|\right\}
\end{aligned}
$$

\section{F. Soft Demapper}

This paper uses soft decoding because LDPC codes has a better performance when uses soft decoding than hard decoding [20]. The $L$ with 4-QAM modulation can be obtained by 


$$
\begin{aligned}
L^{c_{1}} & =\ln \frac{P\left(\chi^{c_{1}=0} \mid \hat{\chi}\right)}{P\left(\chi^{c_{1}=1} \mid \hat{\chi}\right)}, \\
& =\ln \frac{P\left(c_{1}=0, c_{2}=0 \mid \hat{\chi}\right)+P\left(c_{1}=0, c_{2}=1 \mid \hat{\chi}\right)}{P\left(c_{1}=1, c_{2}=0 \mid \hat{\chi}\right)+P\left(c_{1}=1, c_{2}=1 \mid \hat{\chi}\right)}, \\
& =\ln \frac{P\left(\hat{\chi} \mid c_{1}=0, c_{2}=0\right) P\left(c_{1}=0, c_{2}=0\right)+P\left(\hat{\chi} \mid c_{1}=0, c_{2}=1\right) P\left(c_{1}=0, c_{2}=1\right)}{P\left(\hat{\chi} \mid c_{1}=1, c_{2}=0\right) P\left(c_{1}=1, c_{2}=0\right)+P\left(\hat{\chi} \mid c_{1}=1, c_{2}=1\right) P\left(c_{1}=1, c_{2}=1\right)}, \\
& =\ln \frac{P\left(\hat{\chi} \mid c_{1}=0, c_{2}=0\right) P\left(c_{1}=0\right) P\left(c_{2}=0\right)+P\left(\hat{\chi} \mid c_{1}=0, c_{2}=1\right) P\left(c_{1}=0\right) P\left(c_{2}=1\right)}{P\left(\hat{\chi} \mid c_{1}=1, c_{2}=0\right) P\left(c_{1}=1\right) P\left(c_{2}=0\right)+P\left(\hat{\chi} \mid c_{1}=1, c_{2}=1\right) P\left(c_{1}=1\right) P\left(c_{2}=1\right)}, \\
& =\ln \frac{P\left(c_{1}=0\right)\left(P\left(\hat{\chi} \mid c_{1}=0, c_{2}=0\right)+P\left(\hat{\chi} \mid c_{1}=0, c_{2}=1\right) \frac{P\left(c_{2}=1\right)}{P\left(c_{2}=0\right)}\right)}{P\left(c_{1}=1\right)\left(P\left(\hat{\chi} \mid c_{1}=1, c_{2}=0\right)+P\left(\hat{\chi} \mid c_{1}=1, c_{2}=1\right) \frac{P\left(c_{2}=1\right)}{P\left(c_{2}=0\right)}\right)}, \\
& =L_{a}\left(c_{1}\right)+\ln \frac{P\left(\hat{\chi} \mid c_{1}=0, c_{2}=0\right)+P\left(\hat{\chi} \mid c_{1}=0, c_{2}=1\right) e^{-L_{a}\left(c_{2}\right)}}{P\left(\hat{\chi} \mid c_{1}=1, c_{2}=0\right)+P\left(\hat{\chi} \mid c_{1}=1, c_{2}=1\right) e^{-L_{a}\left(c_{2}\right)},}, \\
= & \ln \frac{P\left(\hat{\chi} \mid c_{1}=0, c_{2}=0\right)+P\left(\hat{\chi} \mid c_{1}=0, c_{2}=1\right)}{P\left(\hat{\chi} \mid c_{1}=1, c_{2}=0\right)+P\left(\hat{\chi} \mid c_{1}=1, c_{2}=1\right)}, \\
= & \ln \frac{\exp \left(-\frac{\left(\hat{\chi}-\chi_{a}\right)^{2}}{2 \sigma^{2}}\right)+\exp \left(-\frac{\left(\hat{\chi}-\chi_{b}\right)^{2}}{2 \sigma^{2}}\right)}{\exp \left(-\frac{\left(\hat{\chi}-\chi_{c}\right)^{2}}{2 \sigma^{2}}\right)+\exp \left(-\frac{\left(\hat{\chi}-\chi_{d}\right)^{2}}{2 \sigma^{2}}\right)}, \\
= & \ln \frac{\sum_{i=1}^{2} \exp \left(-\frac{\left(\hat{\chi}-\chi^{c_{i}=0}\right)^{2}}{2 \sigma^{2}}\right)}{\sum_{i=1}^{2} \exp \left(-\frac{\left(\hat{\chi}-\chi^{c_{i}=1}\right)^{2}}{2 \sigma^{2}}\right)},
\end{aligned}
$$

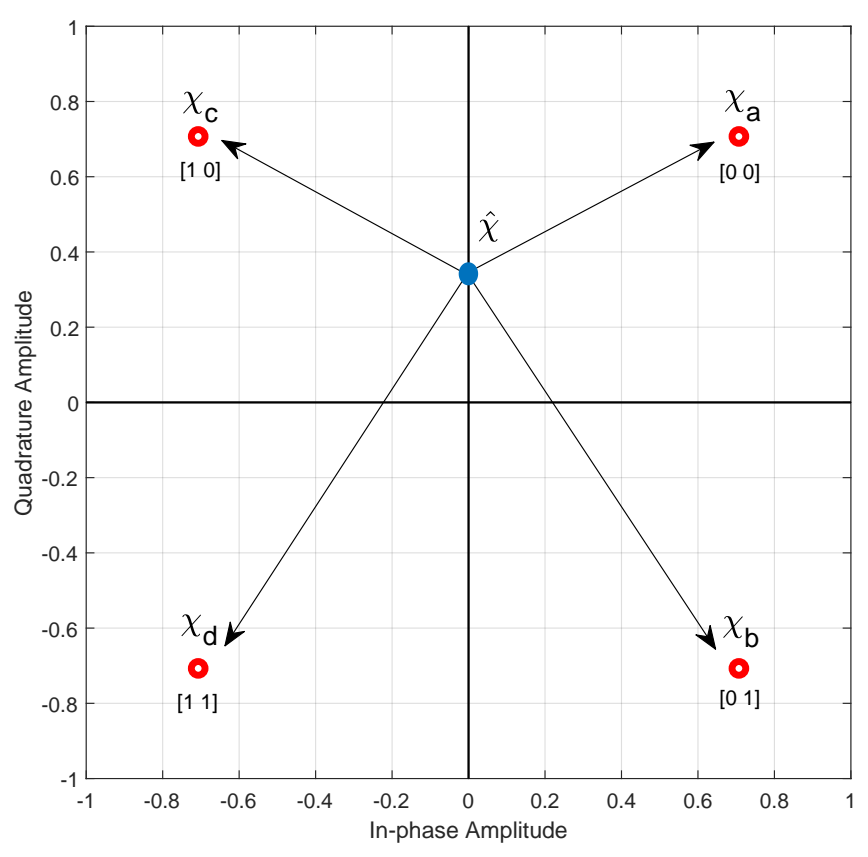

Fig. 7 The condition when $\hat{\chi}$ falls exactly on the quadrant border line.

because $c_{1}$ and $c_{2}$ are independent of (27) due to the use of Gray mapping. In (30), a priori LLR is zero because there is no iterative decoding, then the equation is simpler. Some devices have limitations in performing calculations. This limitation can cause the LLR to be infinity. In MATLAB, the result of exponent of -710 or +710 is infinite. This can cause calculation errors when demapping using soft demap- per. Soft demapper is optimized by minimizing $P_{b}$ [12]

$$
-710<f(\sigma)<710
$$

where $f(\sigma)=\frac{d}{2 \sigma^{2}}$ and $d$ is the distance between $\hat{\chi}$ and the modulation symbol. The smaller $d$ indicates that $\hat{\chi}$ is closer to the modulation symbol. The $\sigma$ is varying depending on the noise and causes $f(\sigma)$ to be infinite when $\sigma \rightarrow 0$.

This can be a problem in (32) if $\hat{\chi}$ falls right on the boundary line because the $\sigma$ approaches 0 which causes infinite LLR, as Fig. 7 shows. In [12], Hamdi et al. researched and proposed the best $d_{p}$ for fifth generation new radio (5G-NR) [12] communication to solve this problem. The $d_{p}$ obtained still has to be optimized, which aims to minimize the $P_{b}$, with the function [12]

$$
-710<\frac{d}{2\left(d_{p}\right)^{2}}<710 .
$$

In [12], the $\sigma$ is $d_{p}$ if $d<d_{p}$. However, the performances using $d_{p}$ have no improvement. This paper proposes threshold $\mathcal{S}$ to solve this problem. Equation (32) is proposed to be changed to

$$
L^{c_{1}}= \begin{cases}\ln \frac{\sum_{i=1}^{2} \exp \left(-\frac{\left(\hat{\boldsymbol{\chi}}-\chi^{c_{i}=0}\right)^{2}}{2(\mathcal{S})^{2}}\right)}{\sum_{i=1}^{2} \exp \left(-\frac{\left(\hat{\boldsymbol{\chi}}-\chi^{c_{i}=1}\right)^{2}}{2(\mathcal{S})^{2}}\right)}, & \text { if } \sigma<\mathcal{S} \\ \ln \frac{\sum_{i=1}^{2} \exp \left(-\frac{\left(\hat{\boldsymbol{\chi}}-\chi^{c_{i}=0}\right)^{2}}{2 \sigma^{2}}\right)}{\sum_{i=1}^{2} \exp \left(-\frac{\left(\hat{\boldsymbol{\chi}}-\chi^{c_{i}=1}\right)^{2}}{2 \sigma^{2}}\right)}, & \text { otherwise. }\end{cases}
$$

This method also applies to $L^{c_{2}}$.

Fig. 8 shows the process of finding the best threshold $\mathcal{S}$ at range $0.0001-1$. Fig. 8 shows that the threshold $\mathcal{S}=\{0.0001,0.001,0.01\}$ experienced an increase in BER at SNR $\gamma=30 \mathrm{~dB}$ and the best performance is at $\mathcal{S}=$ 0.1 . To find out if there is a threshold $\mathcal{S}$ that is better than threshold $\mathcal{S}=0.1$, then an observation for the threshold $\mathcal{S}$ is performed again with a narrowed range, at $0.1-1$ as shown 
TABLE II

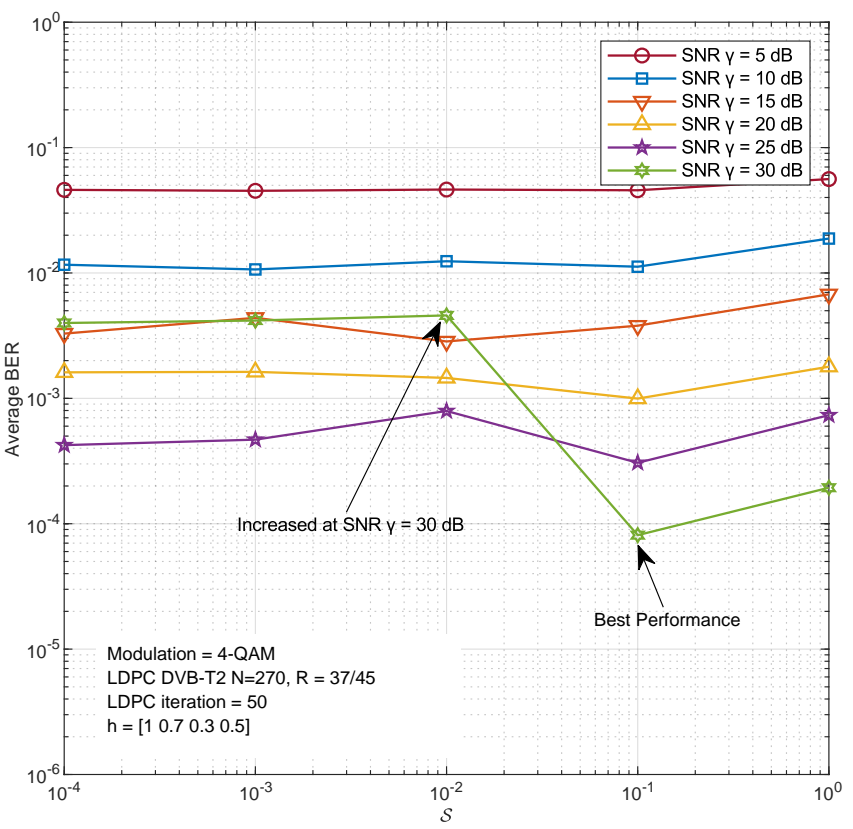

Fig. 8 Observation of threshold $\mathcal{S}$ in range $0.0001-1$.

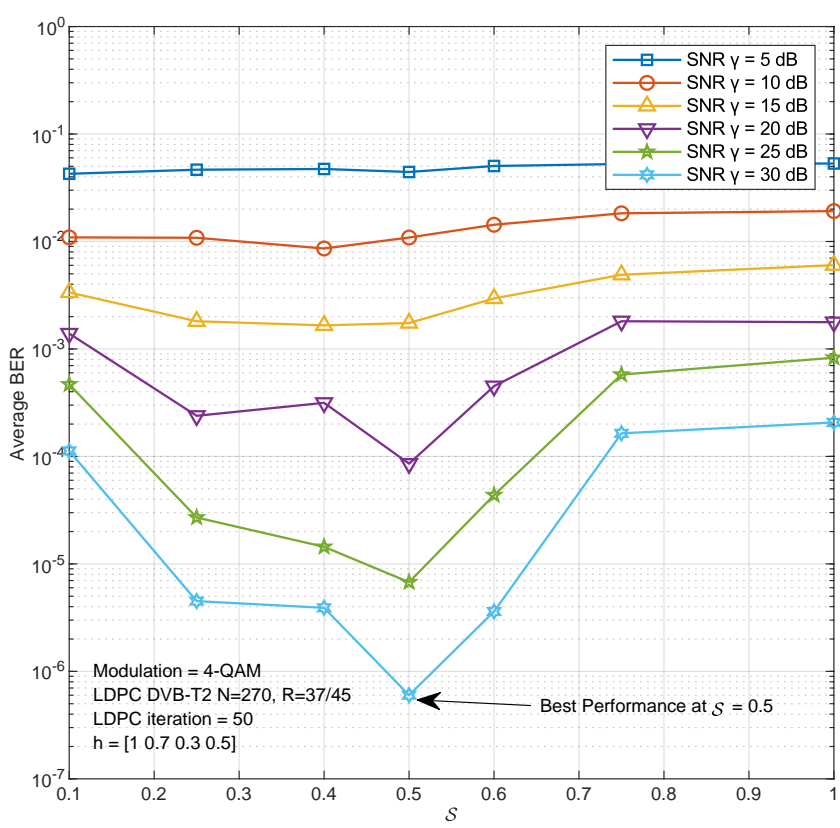

Fig. 9 Observation of threshold $\mathcal{S}$ in range $0.1-1$.

in Fig. 9. Fig. 9 shows that, at threshold $\mathcal{S}=0.5$ has a better performance than the performance of threshold $\mathcal{S}=0.1$. An observation like this applies to all code rates. The results show if the threshold $\mathcal{S}$ cannot be set too small closer to 0 because it causes infinite value of LLR. On the other hand, is the $\mathcal{S}$ value is set too high, e.g. $\mathcal{S}=1$, the demapper just introduce additional errors. Therefore, the optimal threshold
THE PROPOSED VALUES OF THRESHOLD $\mathcal{S}$

\begin{tabular}{cccc}
\hline \multirow{2}{*}{$\operatorname{Re}$} & \multirow{2}{*}{ AWGN } & \multicolumn{2}{c}{ Multipath Rayleigh fading } \\
\cline { 2 - 4 } & & Slow fading & Fast fading \\
\cline { 2 - 4 } & $\mathcal{S}$ & $\mathcal{S}$ & $\mathcal{S}$ \\
\hline Uncoded & - & 0.1 & 0.1 \\
$4 / 9$ & - & 0.5 & 0.875 \\
$3 / 5$ & - & 0.5 & 0.65 \\
$2 / 3$ & - & 0.5 & 0.5 \\
$11 / 15$ & - & 0.5 & 0.5 \\
$7 / 9$ & - & 0.5 & 0.5 \\
$37 / 45$ & - & 0.5 & 0.5 \\
\hline \multicolumn{3}{c}{ TABLE III } \\
& \multicolumn{3}{c}{ PARAMETER SIMULATION }
\end{tabular}

\begin{tabular}{ll}
\hline Parameter & Value \\
\hline Modulation & 4-QAM \\
Frequency Carrier & $2.2 \mathrm{GHz}$ \\
Subcarrier spacing & $15 \mathrm{kHz}$ \\
Maximum Speed & $2450 \mathrm{~km} / \mathrm{h}$ \\
Pilot symbol position & 1 \\
\hline
\end{tabular}

$\mathcal{S}$ should be carefully searched. The threshold $\mathcal{S}$ is needed practically in the field, for example because of the limitations of hardware which cannot calculate exponent above +709 or below -709 . Table II shows the proposed threshold $\mathcal{S}$ in this paper.

\section{RESULT AND DISCUSSION}

\section{A. Performance of LDPC Codes}

Table III shows the parameters used in this paper. We simulate the system at a maximum speed of $2450 \mathrm{~km} / \mathrm{h}$, which means we also look maximum speed of this system can achieve BER $10^{-2}$. We also use only one pilot symbol, which is located at the first position. It aims to test the system in the worst conditions by using only one pilot symbol.

We simulate the performances of DVB-T2 LDPC codes $N=270$ on the AWGN channel, as shown in Fig. 10 . In this figure, the horizontal axis represents the SNR, and the vertical axis represents the BER of transmitted bits. We simulate with maximum SNR $\gamma=10 \mathrm{~dB}$ and maximum LDPC iteration at 50. The $R_{e}=\frac{4}{9}$ has the best performance. To obtained BER performance $10^{-4}, R_{e}=\frac{4}{9}$ at SNR $\gamma=2.615 \mathrm{~dB}$ and $R_{e}=\frac{37}{45}$ at SNR $\gamma=6.7 \mathrm{~dB}$. Systems with the DVB-T2 LDPC codes $N=270$ has a better performance of around $8.375 \mathrm{~dB}$ for $R_{e}=\frac{4}{9}$ and $4.65 \mathrm{~dB}$ for $R_{e}=\frac{37}{45}$ than systems without channel coding at BER $10^{-4}$.

As shown in Fig. 11, we simulate with maximum SNR $\gamma=30 \mathrm{~dB}$ and maximum LDPC iteration at 50 on the multipath Rayleigh slow fading. We assumed the channel model $h=\left[\begin{array}{llll}1 & 0.7 & 0.5 & 0.3\end{array}\right]$. The $R_{e}=\frac{4}{9}$ has the best performance. To obtained BER performance $10^{-4}, R_{e}=\frac{4}{9}$ at SNR $\gamma=12 \mathrm{~dB}$ and $R_{e}=\frac{37}{45}$ at SNR $\gamma=21.5 \mathrm{~dB}$. Systems with the DVB-T2 LDPC codes $N=270$ has a 


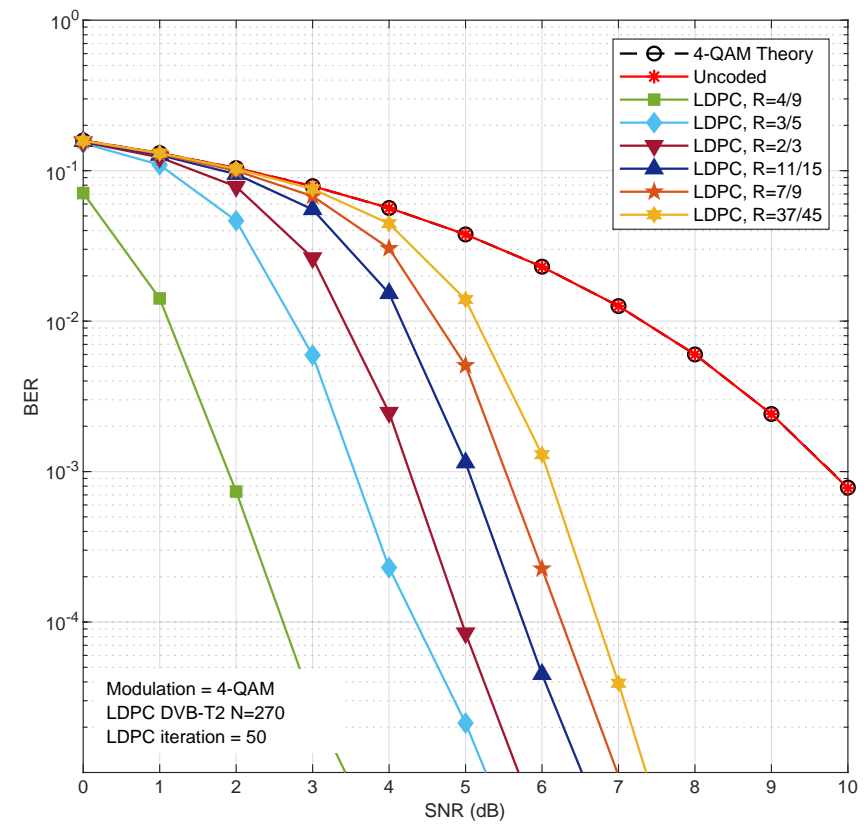

Fig. 10 BER performances of DVB-T2 LDPC codes $N=270$ in AWGN channel.

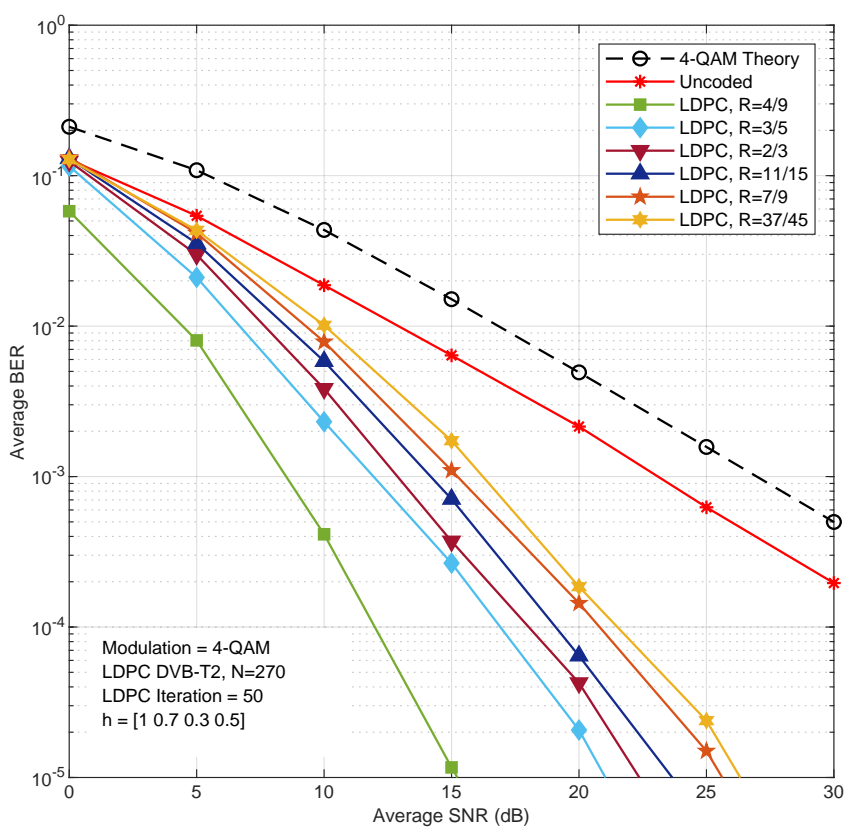

Fig. 11 BER performances of DVB-T2 LDPC codes $N=270$ in multipath Rayleigh slow fading.

better performance of around $21.24 \mathrm{~dB}$ for $R_{e}=\frac{4}{9}$ and $11.74 \mathrm{~dB}$ for $R_{e}=\frac{37}{45}$ than systems without the channel coding at BER $10^{-4}$.

Fig. 12 shows the BER performances against $f_{d} T_{s}$ of the proposed system with DVB-T2 LDPC codes $N=270$. We simulate the system with SNR $\gamma=20 \mathrm{~dB}$ and maximum

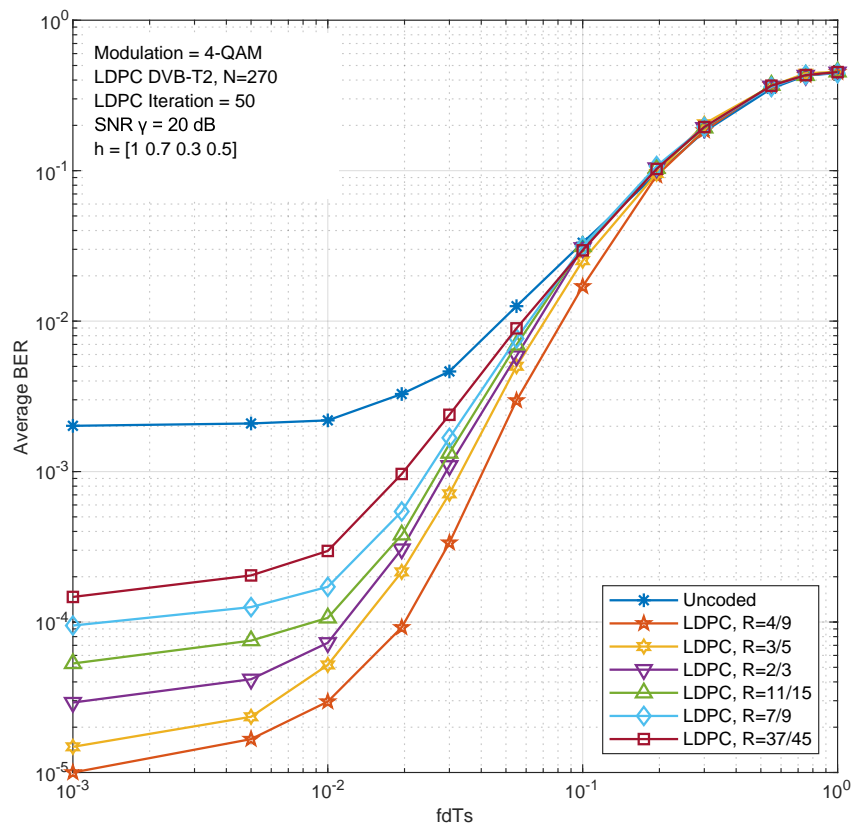

Fig. 12 BER performances against $f_{d} T_{s}$ of the proposed system with DVB-T2 LDPC codes $N_{\mathrm{LDPC}}=270$.

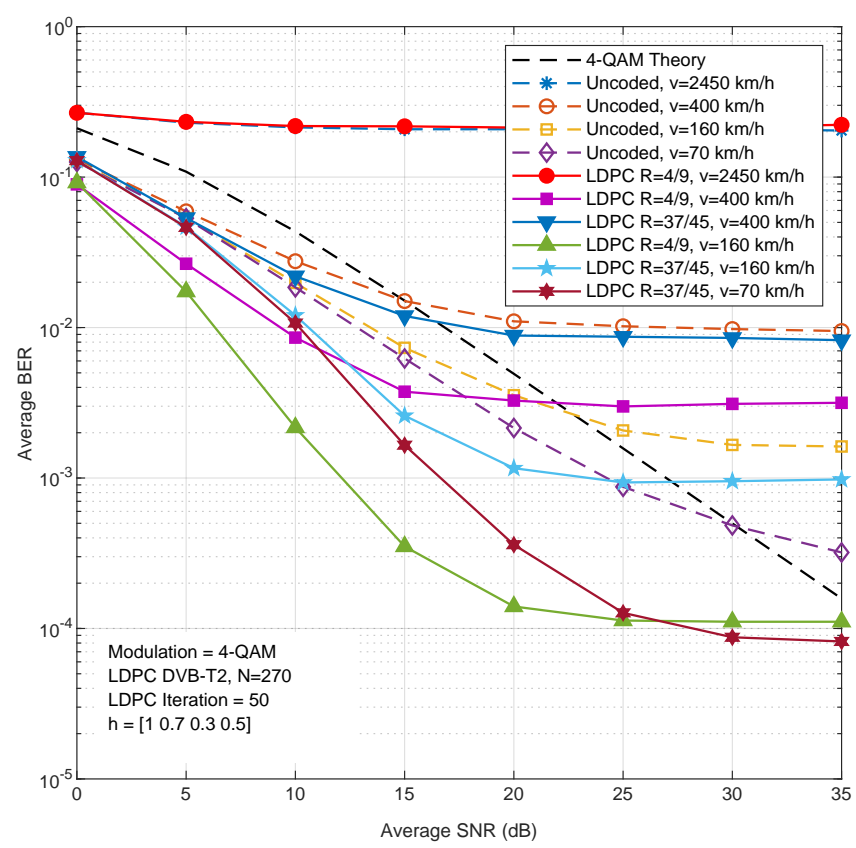

Fig. 13 BER performances of DVB-T2 LDPC codes $N=270$ in multipath Rayleigh fast fading.

LDPC iteration at 50. We assumed the channel model $h=\left[\begin{array}{llll}1 & 0.7 & 0.5 & 0.3\end{array}\right]$. The system without channel coding cannot achieve BER $10^{-2}$ at $f_{d} T_{s}=0.055$. The system with LDPC codes for all code rate can reduce the error and can achieve BER $10^{-2}$ at $f_{d} T_{s}=0.055$. The system with LDPC codes can reduce the error when $f_{d} T_{s} \leq 0.055$. 


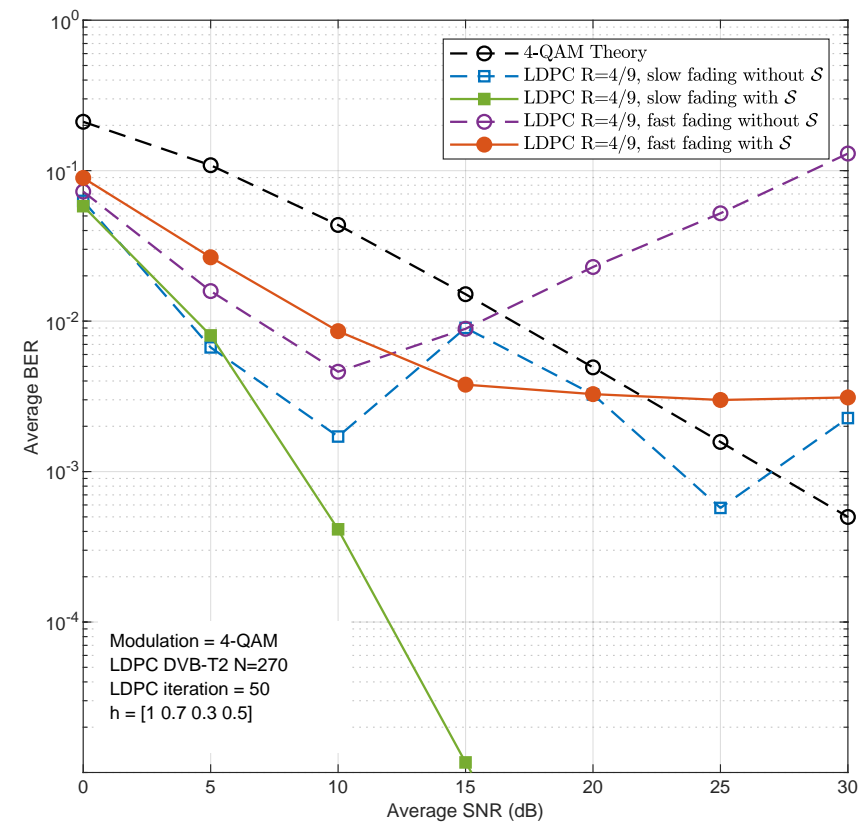

Fig. 14 BER performances of DVB-T2 LDPC codes $N=270$ with $\mathcal{S}$ threshold and without threshold $\mathcal{S}$.

The system with LDPC codes cannot reduce the error when $f_{d} T_{s} \geq 0.195$ and all the code rate have same perfomance with the system without channel coding. The results show channel coding cannot reduce the errors and error-floor if the system is experienced in high speed.

Fig. 13 shows the performances of DVB-T2 LDPC codes $N=270$ on a high-speed flying devices with a maximum speed of $2450 \mathrm{~km} / \mathrm{h}$. We simulate the system with a maximum SNR $\gamma=35 \mathrm{~dB}$ and maximum LDPC iteration at 50. We assumed the channel model $h=\left[\begin{array}{llll}1 & 0.7 & 0.5 & 0.3\end{array}\right]$. When the system experienced at $70 \mathrm{~km} / \mathrm{h}$, the system wihout channel coding cannot achieve BER $10^{-4}$, but LDPC codes with $R_{e}=\frac{37}{45}$ can achieve BER $10^{-4}$ and can reduce the error-floor. When the system experienced at $160 \mathrm{~km} / \mathrm{h}$, the system wihout channel coding cannot achieve BER $10^{-3}$, but LDPC codes with $R_{e}=\frac{4}{9}$ and $R_{e}=\frac{37}{45}$ can achieve BER $10^{-3}$ and can reduce the error-floor. When the system experienced at $400 \mathrm{~km} / \mathrm{h}$, LDPC codes with $R_{e}=\frac{4}{9}$ and $R_{e}=\frac{37}{45}$ can achieve BER $10^{-2}$ and can reduce the errorfloor. Systems with high speed such as $2450 \mathrm{~km} / \mathrm{h}$ have high errors. Even systems with LDPC codes unable to fix errors significantly. The results show channel coding can reduce errors and error-floor when the system is moving with low speed. When the system is moving with high speed, e.g. $2450 \mathrm{~km} / \mathrm{h}$, the channel coding cannot reduce the errors and error-floor.

\section{B. Performance with Threshold $\mathcal{S}$}

Fig. 14 shows the performances of the DVB-T2 LDPC codes $N=270$ with threshold $\mathcal{S}$ and without threshold $\mathcal{S}$ on multipath rayleigh slow fading and fast fading. In fast fading, we use a speed of $v=400 \mathrm{~km} / \mathrm{h}$. The curve of the system without $\mathcal{S}$ threshold is increasing at SNR $\gamma=15 \mathrm{~dB}$ and is unstable in slow fading. In fast fading, the system curve without using $\mathcal{S}$ threshold is increasing at SNR $\gamma=15 \mathrm{~dB}$ and continue to rise without decreasing. This is due to the $\sigma$ is closer to 0 , which produces infinite LLR and causes unstable jumping error.

\section{Conclusion}

This paper has proposed a high-speed flying devices system with the optimal threshold $\mathcal{S}$ for practical applications helped by DVB-T2 LDPC codes such that multimedia transmission from the high-speed flying devices is possible for BER less than $10^{-2}$. This paper confirmed that the DVB-T2 LDPC codes $N_{\text {LDPC }}=270$ can provide good communication performances of flying devices with speed up to $2450 \mathrm{~km} / \mathrm{h}$. The proposed optimal threshold $\mathcal{S}$ with LDPC codes can improve performance and reduce errorfloor at $f_{d} T_{s}$ below 0.055 . The maximum achievable speed that cause error-floor less than $10^{-2}$ is $400 \mathrm{~km} / \mathrm{h}$. This paper has also found that the optimal threshold cannot be set too small $\mathcal{S}<0.1$, because it causes the infinite value of the LLR. On the other hand, if the treshold $\mathcal{S}>0.875$, the demapper just introduce additional errors. Therefore, the optimal threshold $\mathcal{S}$ should be carefully searched. The results of this paper are expected to contribute to the development of communication systems in high-speed flying devices by sending multimedia data in real-time.

\section{REFERENCES}

[1] D. Juniarto, K. Anwar, and D. Arseno, "Communication System for High Speed Flying Devices with Repetition Codes," in Journal of Measurements, Electronics, Communication, and Systems (JMECS), January 2020.

[2] H. Harada and R. Prasad, Simulation and Software Radio for Mobile Communications. USA: Artech House, Inc., 2002.

[3] J. K. Arthur, T. B. T. C. Aka, and A. Acakpovi, "Comparative Analysis of Orthogonal Frequency Division Modulation and Filter BankBased Multicarrier Modulation," in 2019 International Conference on Communications, Signal Processing and Networks (ICCSPN), 2019, pp. $1-10$.

[4] D. Fitriyani, K. Anwar, and D. M. Saputri, "Study on Radio Frequency Profile of Indonesia Digital Television DVB-T2 for Urban Areas." EAI, 12021.

[5] K. Sathananthan and C. Tellambura, "Probability Of Error Calculation Of OFDM Systems With Frequency Offset," Communications, IEEE Transactions on, vol. 49, pp. 1884 - 1888, 122001.

[6] H. Kim, Enhanced Mobile Broadband Communication Systems*, 2020, pp. 239-302.

[7] C. Yang, M. Zhan, Y. Deng, M. Wang, X. H. Luo, and J. Zeng, "Error-correcting Performance Comparison for Polar Codes, LDPC Codes and Convolutional Codes in High-performance Wireless," in 2019 6th International Conference on Information, Cybernetics, and Computational Social Systems (ICCSS), 2019, pp. 258-262.

[8] S. A. Ghauri, M. E. U. Haq, M. Iqbal, and J. U. Rehman, "Performance Analysis of LDPC Codes on Different Channels," in 2014 Eighth International Conference on Next Generation Mobile Apps, Services and Technologies, 2014, pp. 235-240.

[9] K. Arora, J. Singh, and Y. S. Randhawa, "A Survey On Channel Coding Techniques For 5G Wireless Networks," Telecommunication Systems: Modelling, Analysis, Design and Management, vol. 73, no. 4, pp. 637-663, April 2020. [Online]. Available: https://ideas.repec.org/ a/spr/telsys/v73y2020i4d10.1007_s11235-019-00630-3.html

[10] S. Alabady and F. Al-turjman, "Low complexity Parity Check Code for Futuristic Wireless Networks Applications," IEEE Access, vol. 6, pp. 18398-18407, 2018. 
[11] C. Y. Akbar Fadhlika and K. Anwar, "Downscaled LDPC Codes for Indonesia Digital Video Broadcasting Terrestrial 2nd Generation (DVB-T2)," in 2019 Symposium on Future Telecommunication Technologies (SOFTT), vol. 1, 2019, pp. 1-6.

[12] A. Syukra, K. Anwar, and D. M. Saputri, "On the Design of Optimal Soft Demapper for 5G NR Wireless Communication Systems," in 2020 10th Electrical Power, Electronics, Communications, Controls and Informatics Seminar (EECCIS), 2020, pp. 313-318.

[13] 3GPP, "Physical Channels and Modulation," in document 3GPP TS 38.211 version 15.7.0, October 2019, p. 14.

[14] S. J. Johnson, Iterative Error Correction: Turbo, Low-Density ParityCheck and Repeat-Accumulate Codes. Cambridge University Press, 2009.

[15] D. Feng, H. Xu, Q. Zhang, Q. Li, Y. Qu, and B. Bai, "Nonbinary LDPC-Coded Modulation System in High-Speed Mobile Communications," IEEE Access, vol. 6, pp. 50994-51001, 2018.

[16] M. Tomlinson, C. J. Tjhai, M. A. Ambroze, M. Ahmed, and M. Jibril,
Error-Correction Coding and Decoding: Bounds, Codes, Decoders, Analysis and Applications, 1st ed. Springer Publishing Company, Incorporated, 2018.

[17] ETSI, "Digital Video Broadcasting (DVB); Implementation Guidelines For A Second Generation Digital Terrestrial Television Broadcasting System (DVB-T2) ," in ETSI TS 102831 V1.2.1, 2012.

[18] S. Islam, Hasib-Al-Rashid, and M. A. Ullah, "Soft decision multistage threshold decoding with sum-product algorithm," in 2017 8th International Conference on Computing, Communication and Networking Technologies (ICCCNT), 2017, pp. 1-5.

[19] A. Seraj and D. Yadav, "Evaluation of Flexible SPA Based LPDC Decoder Using Hardware Friendly Approximation Methods," 2017, student Paper.

[20] R. Jose and A. Pe, "Analysis of Hard Decision and Soft Decision Decoding Algorithms of LDPC codes in AWGN," in 2015 IEEE International Advance Computing Conference (IACC), 2015, pp. 430435 . 